\title{
Hybrid Technique for Artificial Neural Network Architecture and Weight Optimization
}

\author{
Cleber Zanchettin and Teresa Bernarda Ludermir \\ Center for Informatics, Federal University of Pernambuco (UFPE), \\ P.O. Box 7851, 50.732-970, Recife, PE, Brazil \\ $\{c z$, tbl\}@cin.ufpe.br
}

\begin{abstract}
This work presents a technique that integrates the heuristics tabu search, simulated annealing, genetic algorithms and backpropagation. This approach obtained promising results in the simultaneous optimization of the artificial neural network architecture and weights.
\end{abstract}

\section{Introduction}

Optimization is the process of finding the best solution for a problem from a group of possible solutions. An optimization problem has an objective function and a group of restrictions, both related to the decision variables of the problem. Genetic Algorithms (AG) 3], Simulated Annealing (SA) [1] and Tabu Search (TS) 2] are iterative algorithms used to solve different combinatorial optimization problems. These three algorithms are the most popular from a class of optimization algorithms known as general iterative algorithms. All three optimization heuristics have similarities [4]: (1) They are approximation (heuristic) algorithms, i.e., they do not assure the finding of an optimal solution; (2) They are blind in that they do not know when they have reached an optimal solution, and therefore, must be told when to stop; (3) They have a "hill climbing" property, i.e., they occasionally accept uphill (bad) moves; (4) They are general, i.e., they can easily be engineered to implement any combinatorial optimization problem; all that is required is to have a suitable solution representation, a cost function, and a mechanism to traverse the search space; and (5) Under certain conditions, they asymptotically converge to an optimal solution.

This paper presents a new technique that integrates the main potentialities of these three heuristics. This technique is evaluated in the simultaneous optimization of the number of connections and weight connection values among processing units of the Multi-Layer Perceptron neural network (MLP) [5].

The MLP trained by the backpropagation algorithm (BP) is one of the most used connectionist models in the literature. To obtain successful use, the network topology plays a very important role. A lack of connections can render the network incapable of solving the investigated problem as a result of the inadequacy of adjustable parameters, whereas an excess of connections can cause overfitting in the training data and fail to have an adequate generalization capacity. In general, the training of the MLP neural networks is accomplished through successive attempts 
with different network topologies until reaching satisfactory results for the problem. Besides consuming time, this process can establish network architectures with unnecessary connections and nodes. Moreover, the larger the topology, the more complex the value adjustment of these connections becomes. Thus, the simultaneous optimization of architectures and weights of artificial neural networks is an interesting approach to the generation of efficient networks with small topologies.

\section{Search Heuristics Description}

The genetic algorithm is characterized by a parallel search of the state space as against a point-by-point search through conventional optimization techniques. The parallel search is accomplished by keeping a set of possible solutions for the optimization problem, called population. An individual in the population is a string of symbols and is an abstract representation of the solution. The symbols are called genes and each string of genes is termed a chromosome. The individuals in the population are evaluated through a fitness measure. The population of chromosomes evolves from one generation to the next through the use of two types of genetic operators: (1) unary operators, such as mutation and inversion, which alter the genetic structure of a single chromosome; and (2) higher-order operator, referred to as crossover, which consists of obtaining a new individual by combining genetic material from two selected parent chromosomes. The parent chromosomes are chosen by way of selection techniques 3 .

In the experiments performed, each chromosome is represented as described in Section 3. The initial population was defined with a size of 10 chromosomes. The chromosomes are classified by Rank Based Fitness Scaling [8]. The parents chosen for the next generation is accomplished in a probabilistic manner, using Universal Stochastic Sampling [8]. Elitism was also used, with a probability of $10 \%$. For the combination of the parent chromosomes, the crossover operator Uniform Crossover [9] was used, with a probability of $80 \%$. The mutation operator used was the Gaussian Mutation [6], with a probability of $10 \%$. The stop criteria were: (1) the $G L_{5}$ criterion, this criterion provides an idea of the generalization loss during training and it is sufficiently useful to avoid overfitting. It is defined as the increase in the validation error in relation to the minimum validation error; and (2) a maximum number of 500 generations.

The simulated annealing method is different of the others search methods in that uphill moves are occasionally accepted to escape of local minima. The search process consists of a sequence of iterations. Each iteration consists of randomly changing the current solution to create a new solution in its neighborhood. Once a new solution is created, the corresponding change in the cost function is computed to decide if the new solution can be accepted. If the new solution cost is lower than the current solution cost, is accepted. Otherwise, the Metropolis's criterion is verified [10, based on the Boltzmann probability. A random number $d$ in $[0,1]$ interval is generated from a uniform distribution. If $\delta \leq e^{\frac{\Delta E}{T}}$, where $\Delta E$ is the change in the cost function and $T$ is a parameter called temperature, then the new solution is accepted as the current solution. If not, the current solution is unchanged and the process continues from the current solution. 
The algorithm was originally derived from thermodynamic simulations. Thus, the parameter $T$ is referenced as temperature and the temperature reduction process is called the cooling process. The chosen cooling strategy was geometric cooling rule. According to this rule, the new temperature is equal to the current temperature multiplied by a temperature factor (smaller than one, but close to one) [1]. The initial temperature is set to 1 , and the temperature factor is set to 0.9 . The temperature is decreased at each 10 iterations, with a maximum number of 1.000 iterations. The stop criterion $G L_{5}$ also was used.

Tabu search is an iterative search algorithm characterized by the use of a flexible memory. In this method, each iteration consists of the evaluation of a certain amount of new solutions (neighborhood moves). The best of these solutions (in terms of cost function) is accepted. However, the best candidate solution may not improve the current solution. Thus, the algorithm chooses the new solution that produces the largest improvement or the smallest deterioration in the cost function. This strategy allows the method to escape from local minima. A tabu list is used to store a certain amount of recently visited solutions. The solutions in tabu list are marked as forbidden to subsequent iterations. The tabu list registers $T$ last visited solutions. When the list is full, a new movement is registered in substitution to the older solution kept on the list.

In the present work, a neighborhood with 20 solutions is used, and the algorithm chooses the best non-tabu solution. The proximity criterion [6] was used to compare solutions. A new solution is considered identical to the tabu solution one if: (1) each connectivity bit in the new solution is identical to the corresponding connectivity bit in the tabu solution; and (2) each connection weight in the new solution is within $\pm N$ of the corresponding connection weight in the tabu solution. The parameter $N$ is a real number with a value of 0.001 . A maximum number of 100 iterations is allowed. The stop criterion employed was also $G L_{5}$.

\section{Integration of Simulated Annealing, Tabu Search and Genetic Algorithms}

The simulated annealing method has the ability to escape from local minima through the choice between accepting or discarding a new solution that increases cost (uphill moves). The tabu search method, in contrast, evaluates one group of new solutions at each iteration (instead of only one solution as in simulated annealing). This makes a tabu search faster, as it generally needs less iterations to converge. The genetic algorithm evolution, in turn, involves a sequence of iterations, where a group of solutions evolves through selection processes and reproduction. This process, which is more elaborate than the other algorithms, can result in solutions with a better quality.

These observations motivated the proposal of an optimization technique (GaTSa) that combines the main potentialities of genetic algorithms, simulated annealing and tabu search in an effort to avoid their limitations. In general terms: at each iteration, a group of new solutions is generated, starting from the micro-evolution of the current population, as in genetic algorithms. The cost of 
each solution is evaluated, and the best solution is chosen, as in tabu search. However, differently from a tabu search, this solution is not always accepted. The acceptance criterion is the same used in the simulated annealing algorithm - if the chosen solution has a smaller cost than the current solution, it is accepted; otherwise, it can either be accepted or not, depending on a probability calculation. This probability is given by the same expression used in the simulated annealing method. The visited solutions are marked as tabu, as in a tabu search. During the optimization process, only the best solution found is stored, that is, the final solution comes back through the method.

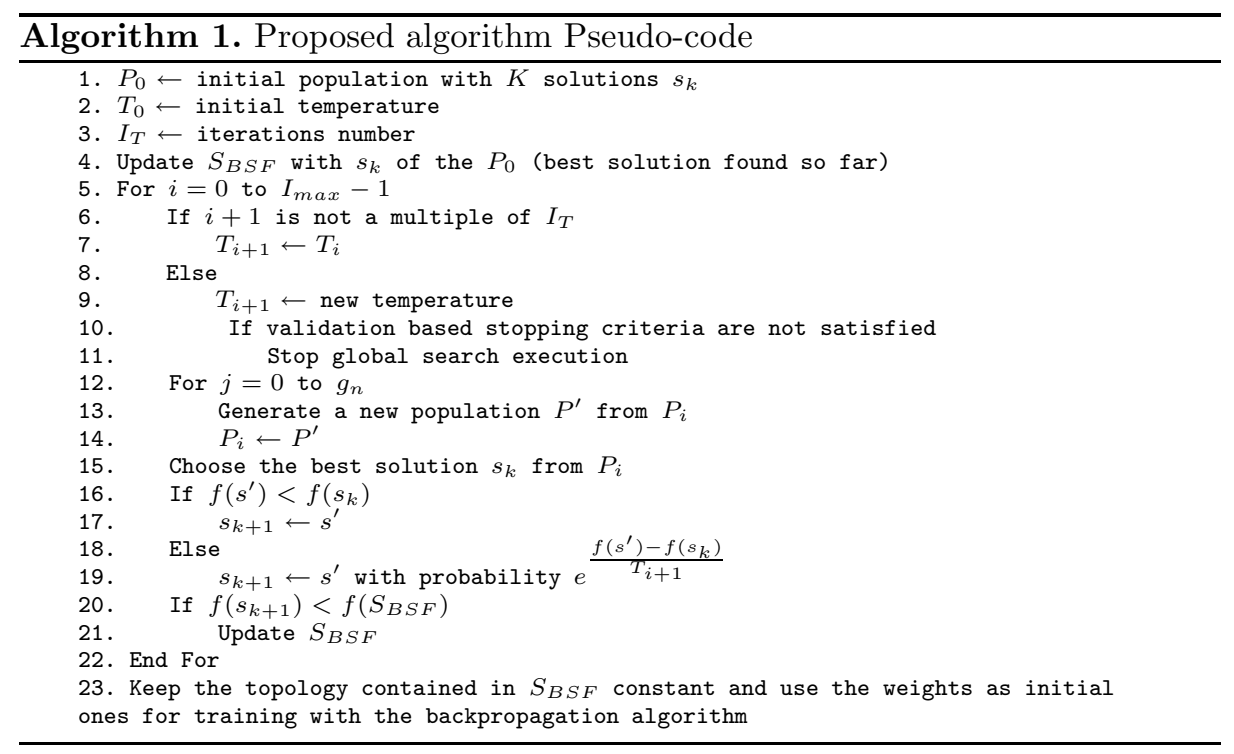

The proposed method pseudo-code is presented in Algorithm 1. Let $S$ be a group of solutions and $f$ a real cost function, the proposed algorithm searches the global minimum $s$, such that $f(s) \leq f\left(s^{\prime}\right), \forall s^{\prime} \in S$. The process finishes after $I_{\max }$ iterations or if the stop criterion based on the validation error is satisfied. The best found solution $S_{B S F}$ (best so far) is returned. The cooling process updates the temperature $T_{i}$ of the iteration $i$ at each $I_{T}$ algorithm iterations. At each iteration, a new population with $k$ solutions is generated. A genetic micro-evolution of $g_{n}$ generations is used to generate this population from the current population. Moreover, at the end of the global search (GaTSa), a hybrid training is used, combining the proposed method with a local search technique. The local search technique can be implemented, for instance, by the well-known backpropagation algorithm.

Each solution is codified in a vector. This vector represents the connections among the processing units of the MLP artificial neural network. Each of these connections is specified by two parameters: (a) the connectivity bit, a boolean value that simbolizes the existence or absence of a connection; and (b) the connection weight, which is a real number. If the connectivity bit is equal to zero, 
its associated weight is not considered, for the connection does not exist in the network. All possible connections among adjacent layers are considered.

Different from the constructive algorithms that only generate one solution at the end of the process, iterative algorithms originate possible (candidate) solutions at each iteration. The cost function is used to evaluate the performance among consecutive iterations and select the solution that minimizes (or maximizes) an objective function.

The cost function for the investigated problem is the arithmetic average between: (1) the classification error of the training set (percentage of incorrectly classified training patterns); and (2) the percentage of connections used by the artificial neural network. Therefore, the algorithms try to minimize both network performance and processing complexity. Only valid networks (i.e., networks with at least one unit in the hidden layer) were considered.

The operator for the generation of neighbors is used to derive new solutions from the current solution. The method used in simulations is defined as follows: (1) the connectivity bits for the current solution are changed according to a given probability, which in the present work is set to $20 \%$. This operation deletes some network connections and creates new ones. Next, a random number taken from a uniform distribution in $[-1 ;+1]$ is added to each connection weight. These two steps can change both topology and connection weights to produce a new neighbor solution.

\section{Experiments and Results}

Real data is used in the experiments. The problem aims to classify odor patterns obtained through an artificial nose. The odorant compositions analyzed are from three different vintages (years 1995, 1996 and 1997) of the same commercial red wine (Almadm, Brazil) produced with merlot-type grapes. The artificial nose used is composed of six distinct conducting polymer sensors constructed with an electrochemical deposition of polypyrrole using different types of dopants. Three data acquisitions were performed. In each acquisition for each wine vintage, the resistance value of each sensor was recorded for five seconds. A set of six values from the six sensors at the same time was considered a pattern. Thus, each acquisition contains 1.800 patterns (600 from each vintage). There were three acquisitions and 5.400 patterns of data.

In previous works with this data base, the best performance obtained by the MLP was achieved by an architecture with 6 processing units in the input layer, 4 processing units in the hidden layer and 3 processing units in the output layer [7. This topology was keep constant as the maximum architecture in the optimization experiments performed. In all investigated algorithms, the parameter configurations were maintained at the standard configuration or adjusted based on previous experiments. The values used may not be the best values for the problem, but the objective of the present paper is to demonstrate the potentialities of the techniques and not the ideal algorithms configuration.

Table 1 presents the average performance of each investigated optimization technique. These results were obtained for each technique in the optimization 
of the number of connections and weight connection values of an MLP artificial neural network. The parameters evaluated were: (1) Squared Error Percentage (SEP) and the classification error (Class) of training, validation and test sets; (2) algorithm iteration number; (3) artificial neural network connection number; and (4) the temperature value. The following table displays the average results of 10 simulations. Each simulation contains 30 different runs of the algorithm.

Table 1. Optimization techniques performance

\begin{tabular}{|c|c|c|c|c|c|c|}
\hline \multirow[b]{2}{*}{ Technicque } & Training & Validation & Test & \multirow[b]{2}{*}{ Iterations } & \multirow[b]{2}{*}{ Connections } & \multirow[b]{2}{*}{ Temperature } \\
\hline & SEP Class & SEP Class & SEP Class & & & \\
\hline $\mathrm{TS}$ & $18,74 \quad 5,44$ & $18,86 \quad 5,88$ & $18,75 \quad 5,3805$ & 51 & 11,42 & - \\
\hline SA & $19,65 \quad 6,91$ & $19,76 \quad 7,47$ & $19,65 \quad 6,9331$ & 715 & 11,77 & 0,0085 \\
\hline GA & $21,6615,88$ & $21,7316,52$ & $21,6615,9240$ & 315 & 16,64 & - \\
\hline GaTSa & $18,693,58$ & $18,76 \quad 3,81$ & $18,693,5664$ & 46 & 8,33 & 0,7098 \\
\hline $\mathrm{GaTSa}+\mathrm{BP}$ & $4,78 \quad-$ & $2,41 \quad-$ & $2,14 \quad 2,8684$ & 86 & 8,33 & 0,7098 \\
\hline $\mathrm{BP}$ & 6,25 & 3,15 & $2,84 \quad 6,7854$ & 90 & 36 & - \\
\hline
\end{tabular}

The technique that combines the heuristics of tabu search, simulated annealing and genetic algorithms obtained the best result performance. This technique was better even without using the local search heuristic to optimize the artificial neural network connection values. The average classification error obtained was $2.87 \%$, with an average of 8 connections from 36 possible connections in a fully connected neural network. Using a full connected network, the local optimization technique backpropagation obtained an average error of $6.78 \%$.

The genetic algorithms, tabu search and simulated annealing methods incorporate domain specific knowledge in their own search heuristics. They also tolerate some elements of non-determinism, which helps the search escape from local minima. They rely on the use of a suitable cost function that provides feedback to the algorithm as the search progresses. The main difference among them is how and where domain-specific knowledge is used. For example, in simulated annealing such knowledge is mainly included in the cost function. Solutions involved in a perturbation are selected randomly, and perturbations are accepted or rejected according to a probability.

In the case of genetic algorithms, domain specific knowledge is exploited in all phases. The fitness of individual solutions, the reproduction selection, genetic operators, as well as the generation of the new population, incorporate domainspecific knowledge. Tabu search is different from the above heuristics in that it has an explicit memory component. At each iteration, the neighborhood of the current solution is partially explored, and a move is made to the best non-tabu solution in that neighborhood. The neighborhood function, together with the size and content of the tabu list, is problem specific. The direction of the search is also influenced by memory structures.

The proposed integration uses a larger amount of information on the problem domain and uses this information in practically all search phases. This is possible through the integration of the main potentialities of the three investigated search 


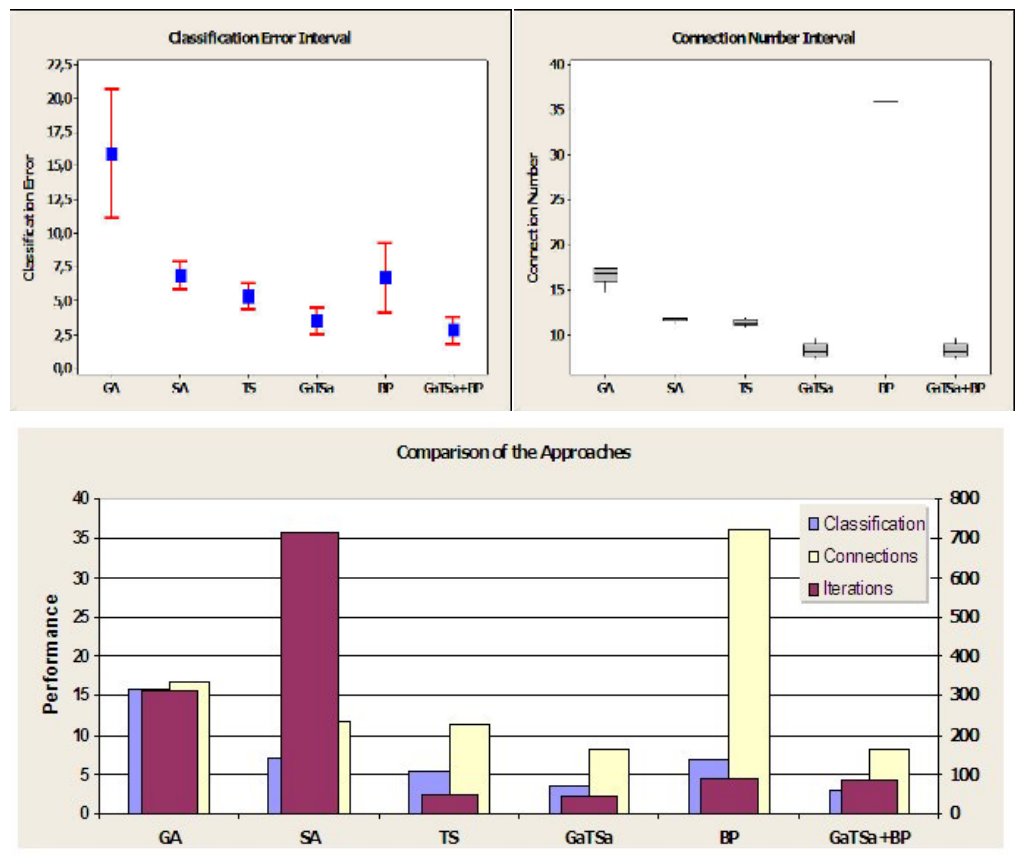

Fig. 1. Result analysis

heuristics. Moreover, the proposed technique has two well-defined stages: a global search phase, where it makes use of the capacity for generating new solutions for the genetic algorithms, the cooling process and cost function of the simulated annealing as well as the memory characteristics of the tabu search technique; and a local search phase, where it makes use of characteristics such as gradient descending for a more precise solution adjustment. These characteristics can obtain better solutions for the investigated problems, with a short search time, low computational cost and minimal investigated search space.

Figure 1 presents graphs comparing the performance of the investigated techniques. The proposed technique obtained the best results regarding the classification error, final network connection number and the number of iterations needed for architecture optimization.

\section{Final Remarks}

This work presented a technique that integrates the heuristics of tabu search, simulated annealing, genetic algorithms and backpropagation. In the simultaneous optimization of the connection number and connection values of the MultiLayer Perceptron neural network, this technique obtained promising results in comparison with the isolated techniques. The proposed technique combines strategies of global and local searches, presenting promising results regarding 
the investigated solution space, computacional cost and search time. The investigated problem involves a critical subject, the stability versus plasticity relation in the training of artificial neural networks.

Without a deeper investigation, it is not possible to say if these results can be extended to other problem classes. An interesting theoretical study proved a number of theorems stating that the average performance of any pair of iterative (deterministic or non-deterministic) algorithms across all problems is identical. Thus, if an algorithm performs well on a certain class of investigated problems, it necessarily pays for that with degraded performance on the remaining set of problems [12]. Future investigations should consider this presupposition and verify the performance of this optimization technique on other problems.

\section{Acknowledgments}

The authors would like to thank CNPq, CAPES and FINEP (Brazilian research agencies) for their financial support.

\section{References}

1. Kirkpatrick, S., Gellat Jr, C. D., Vecchi, M. P.: Optimization by simulated annealing. Science 220 (1983) 671-680

2. Glover, F.: Future paths for integer programming and links to artificial intelligence. Computers and Operation Research 13 (1986) 533-549

3. Goldberg, D. E.: Genetic Algorithms in Search, Optimization and Machine Learning. Addison-Wesley Longman Publishing Co., Inc. (1989) 372 pages

4. Sait, S. M., Youssef, H.: Iterative Computer Algorithms with Applications in Engineering: Solving Combinatorial Optimization Problems. IEEE Computer Society Press (1999) 387 pages

5. Rumelhart, D. E., Hilton, G. E., Williams, R. J.: Learning Representations by Backpropagation Errors. Nature 323 (1986) 533-536

6. Sexton, R J., Alidaee, B., Dorsey, R. E., Johnson, J. D.: Global Optimization for Artificial Neural Networks: A Tabu Search Application. European Journal of Operational Research 106:2-3 (1998) 570-584

7. Yamazaki, A., de Souto, M.C.P., Ludermir, T.B.: Optimization of Neural Network Weights and Architectures for Odor Recognition using Simulated Annealing. In Proceedings International Joint Conference on Neural Networks (2002) 547-552

8. Baker, J. E.: Reducing bias and inefficiency in the selection algorithm. In Proceedings of the Second International Conference on Genetic Algorithms and their application. Lawrence Erlbaum Associates (1987) 14-21

9. Sywerda, G.: Uniform crossover in genetic algorithms. In Proceedings of international conf. on Genetic algorithms. Morgan Kaufmann Publishers Inc. (1989) 2-9

10. Metropolis, N., Rosenbluth, A. W., Rosenbluth, M. N., Teller, A. H., Teller, E.: Equation of state calculations by fast computing machines. The Journal of Chemical Physics 21:6 1087-1092

11. Pham, D. T., Karaboga, D.: Intelligent Optimisation Techniques. Springer-Verlag New York (1998) 312 pages

12. Wolpert, D. H., Macready, W. G.: No free lunch theorems for optimization. IEEE Transactions on Evolutionary Computation 1:1 (1997) 67-82 